\title{
Dynamics of excitons in individual InAs quantum dots revealed in four-wave mixing spectroscopy: supplementary material
}

\author{
Q. Mermillod ${ }^{1,2}$, D. WigGeR ${ }^{3}$, V. Delmonte ${ }^{1,2}$, D. E. ReiteR ${ }^{3}$,

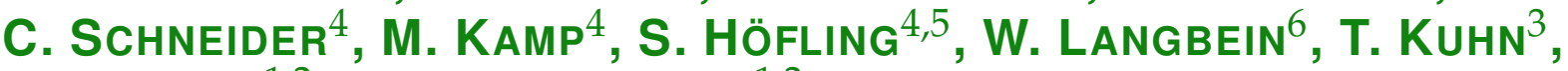 \\ G. NOGUES ${ }^{1,2}$, AND J. KASPRZAK ${ }^{1,2}$ \\ 1 Univ. Grenoble Alpes, F-38000 Grenoble, France \\ ${ }^{2}$ CNRS, Institut Néel, "Nanophysique et semiconducteurs" group, F-38000 Grenoble, France \\ ${ }^{3}$ Institut für Festkörpertheorie, Universität Münster, 48149 Münster, Germany \\ ${ }^{4}$ Technische Physik and Wilhelm Conrad Röntgen Research Center for Complex Material Systems, Universität Würzburg, Germany \\ ${ }^{5}$ SUPA, School of Physics and Astronomy, University of St Andrews, St Andrews, KY16 9SS, United Kingdom \\ ${ }^{6}$ Cardiff University School of Physics and Astronomy, The Parade, Cardiff CF24 3AA, United Kingdom \\ *Corresponding author: jacek.kasprzak@neel.cnrs.fr
}

Published 1 April 2016

\begin{abstract}
This document provides supplementary information to the research article entitled "Dynamics of excitons in individual InAs quantum dots revealed in four-wave mixing spectroscopy," http://dx.doi.org/10.1364/optica.3.000377. (C) 2016 Optical Society of America
\end{abstract}

http://dx.doi.org/10.1364/optica.3.000377.s001

\section{SAMPLE PREPARATION AND CHARACTERIZATION}

The MBE grown sample contains a layer of annealed and capped InAs QDs with a nominal density of $2.2 \times 10^{9} \mathrm{~cm}^{-2}$. They are embedded in an asymmetric GaAs/AlGaAs micro-cavity exhibiting a low quality factor [1-4] $Q=170$, resulting in a mode centered at $910-915 \mathrm{~nm}$ with a FWHM of around $10 \mathrm{~nm}$. The femto-second laser pulse trains are spectrally matched with such a large spectral window and efficiently penetrate into the structure. Furthermore, the intra-cavity field is enhanced by a factor of $\sqrt{Q}=13$ improving the coupling between $E_{1,2,3}$ and the electric dipole moment $\mu$ of the transition. Thus, the resonant field required to drive the FWM is reduced by a factor $Q^{3 / 2} \simeq 2200$ and the signal-to-noise ratio of the interferometrically detected FWM is amplified accordingly.

The sample is intentionally doped with $\mathrm{Si}(\delta$-doping with a nominal density of $1.8 \times 10^{10} \mathrm{~cm}^{-2}$; layer located $10 \mathrm{~nm}$ below the QD plane). To identify the spatial and spectral location of the QD transitions we perform hyperspectral imaging $[5,6]$. In Fig. S1 a we present an example of such imaging performed in a confocal micro-photoluminescence (PL) experiment. Each bright spot corresponds to a QD emission, primarily attributed to recombination of negative trions $\left(\mathrm{GX}^{-}\right)$due to the $n$-doping. We detect high PL counting rates on the order of $10^{5} / \mathrm{sec}$ at the QD saturation. Such an unusually bright PL emission is attributed to the presence of oval photonic defects on the sample surface [3,4], acting as natural micro-lenses [7]. Additionally, the inhomogeneous broadening due to spectral wandering is largely reduced $[1,4]$ indicating an excellent structural quality of these QDs.

The FWM hyperspectral imaging at the same sample area and spectral range is shown in Fig. S1 b. The three QDs at $(x, y) \approx(-2 \mu \mathrm{m},-5 \mu \mathrm{m}),(2 \mu \mathrm{m},-5 \mu \mathrm{m})$ and $(7 \mu \mathrm{m},-4 \mu \mathrm{m})$ (marked with green boxes in Fig. S1) exhibit both PL and FWM signals and were used to align the figures. However, other QDs show different distribution of the peak heights in FWM as compared to the PL. This is expected from the different properties determining the signal strength in both measurements: in FWM the dipole moment is probed, while in PL generally the more complex phonon-assisted carrier relaxation combined with a capture of the exciton also lead to a signal. To demonstrate the high spectral and spatial selectivity of the FWM compared to the PL, Figs. S1 c and d compare both PL and FWM obtained from the same sample spot, defined by the diffraction limited size $(0.7 \mu \mathrm{m})$ of the excitation laser. In Fig. S1 c we show a neutral exciton complex, which is only present in few \% of the QDs. The exciton-biexciton system is straightforwardly recognized 

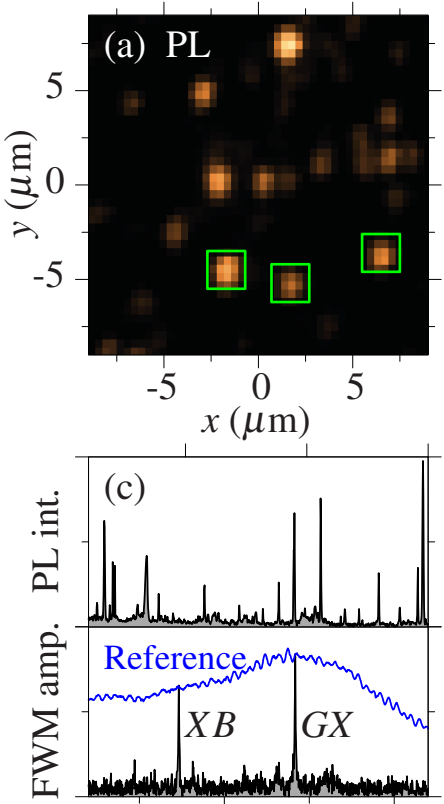

1360 photon energy (meV)

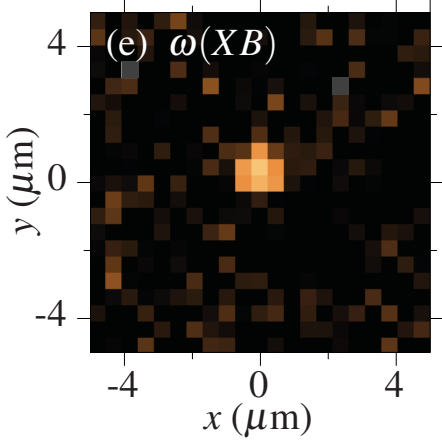

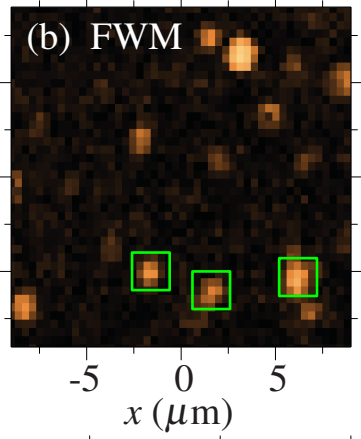
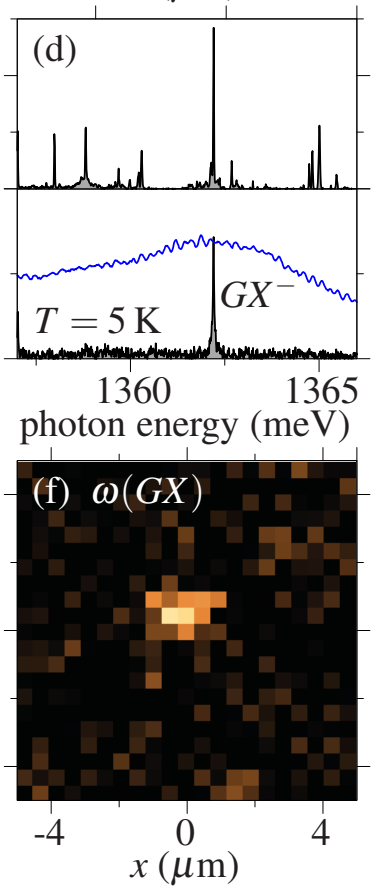

Fig. S1. Sample characterization. Hyperspectral image of the sample for (a) PL and (b) FWM measurements. (c,d) PL and FWM spectra of a single QD. Blue indicates the spectrum of the laser pulse as reference. $(\mathbf{e}, \mathbf{f})$ Hyperspectral FWM image of of $X B$ and $G X$ transition.

in FWM, but it is difficult to be identified in PL, because of a lacking $X B$ emission line. Figure $\mathrm{S} 1 \mathrm{~d}$ shows the PL and FWM spectra of the fundamental trion line corresponding to a negatively charged QD. A zoom-in of the spatial shape of the $X B$ and GX transition is shown in Fig. S1 e and f, respectively.

\section{THEORETICAL MODEL}

The Hamiltonian for circularly polarized excitation (cf. Fig. 1 a) reads

$$
H=\sum_{v} \hbar \omega_{v}|v\rangle\left\langle v\left|-\sum_{v, v^{\prime}} \hbar M_{v v^{\prime}}\right| v\right\rangle\left\langle v^{\prime}\right|+H_{\mathrm{exc}}
$$

with the basis states

$$
|v\rangle \in\left\{|G\rangle,\left|\sigma^{-}\right\rangle,\left|\sigma^{+}\right\rangle,|B\rangle\right\} .
$$

Correspondingly the energies are $\hbar \omega_{G}=0, \hbar \omega_{\sigma^{-}}=\hbar \omega_{\sigma^{+}}=$ $\hbar \omega_{\sigma}$ and $\hbar \omega_{B}=2 \hbar \omega_{\sigma}-\Delta$, where $\Delta$ is the BBE. Due to the Coulomb exchange interaction the two single exciton levels interact via

$$
H_{\mathrm{exc}}=\frac{\delta}{2}\left(\left|\sigma^{-}\right\rangle\left\langle\sigma^{+}|+| \sigma^{+}\right\rangle\left\langle\sigma^{-}\right|\right)
$$

The light field coupling

$$
\underline{M}=\left(\begin{array}{cccc}
0 & \Omega_{\sigma^{+}}^{*} & \Omega_{\sigma^{-}}^{*} & 0 \\
\Omega_{\sigma^{+}} & 0 & 0 & \Omega_{\sigma^{-}}^{*} \\
\Omega_{\sigma^{-}} & 0 & 0 & \Omega_{\sigma^{+}}^{*} \\
0 & \Omega_{\sigma^{-}} & \Omega_{\sigma^{+}} & 0
\end{array}\right)
$$

describes the allowed transitions via the Rabi frequencies

$$
\Omega_{\sigma^{ \pm}}=\sum_{j} \frac{M_{0}}{\hbar} \mathbf{E}_{j} \cdot \mathbf{e}_{\sigma^{ \pm}}^{*} .
$$

$M_{0}$ is the bulk dipole matrix element and $\mathbf{e}_{\sigma^{ \pm}}$the polarization vector. The polarization of the system is given by

$$
\begin{array}{ll}
\mathbf{p}=\quad & M_{0}\left(|0\rangle\left\langle\sigma^{+}|+| \sigma^{-}\right\rangle\langle B|\right) \mathbf{e}_{\sigma^{+}} \\
+ & M_{0}\left(|0\rangle\left\langle\sigma^{-}|+| \sigma^{+}\right\rangle\langle B|\right) \mathbf{e}_{\sigma^{-}} .
\end{array}
$$

This Hamiltonian can be transformed into the basis for linearly polarized excitons (cf. Fig. $1 \mathrm{~b}$ ), which are the eigenstates of $\sum_{v} \hbar \omega_{v}|v\rangle\langle v|+H_{\text {exc }}$. The transformation is calculated by

$$
\begin{aligned}
& |X\rangle=\frac{1}{\sqrt{2}}\left(\left|\sigma^{+}\right\rangle+\left|\sigma^{-}\right\rangle\right), \\
& |Y\rangle=\frac{i}{\sqrt{2}}\left(\left|\sigma^{+}\right\rangle-\left|\sigma^{-}\right\rangle\right) .
\end{aligned}
$$

By the diagonalization the degeneracy of the single excitons is lifted and the exciton energies are $\hbar \omega_{X}=\hbar \omega_{\sigma}-\delta / 2$ and $\hbar \omega_{Y}=\hbar \omega_{\sigma}+\delta / 2$. The light field coupling changes to

$$
\underline{M}=\left(\begin{array}{cccc}
0 & \Omega_{X}^{*} & \Omega_{Y}^{*} & 0 \\
\Omega_{X} & 0 & 0 & \Omega_{X}^{*} \\
\Omega_{Y} & 0 & 0 & \Omega_{Y}^{*} \\
0 & \Omega_{X} & \Omega_{Y} & 0
\end{array}\right)
$$

with

$$
\Omega_{X}=\frac{1}{\sqrt{2}}\left(\Omega_{\sigma^{+}}+\Omega_{\sigma^{-}}\right), \quad \Omega_{Y}=\frac{i}{\sqrt{2}}\left(\Omega_{\sigma^{+}}-\Omega_{\sigma^{-}}\right) .
$$

The time evolution of the density matrix $\varrho$ is calculated assuming a sum of $\delta$-pulses yielding the Rabi frequencies for circular polarization

$$
\Omega_{\sigma^{ \pm}}=\sum_{j} \frac{\theta_{j}^{\sigma^{ \pm}}}{2} e^{i \varphi_{j}^{\sigma^{ \pm}}} \delta\left(t-t_{j}\right)
$$

with arrival times $t_{j}$, pulse areas $\theta_{j}^{\sigma^{ \pm}}$and phases $\varphi_{j}^{\sigma^{ \pm}}$. For a pulse sequence with linear polarizations $\alpha_{j}$ with respect to $X$ and pulse areas $\theta_{j}$ the Rabi frequencies read

$$
\begin{aligned}
& \Omega_{X}=\sum_{j} \sqrt{2} \theta_{j} e^{i \phi_{j}} \cos \left(\alpha_{j}\right), \\
& \Omega_{Y}=\sum_{j}-\sqrt{2} \theta_{j} e^{i \phi_{j}} \sin \left(\alpha_{j}\right) .
\end{aligned}
$$

In the case of $\delta$-pulses the time evolution of the system can be calculated by matrix multiplication [8]. In between the pulses the dynamics is given by

$$
\rho_{v v^{\prime}}(t)=\rho_{v v^{\prime}}(0) e^{i \Lambda_{v v^{\prime}}(t)}
$$


with

$$
\begin{aligned}
\Lambda_{v v^{\prime}}= & \omega_{v}-\omega_{v^{\prime}}+i \beta_{v v^{\prime}}, \\
\underline{\beta} & =\left(\begin{array}{cccc}
0 & \beta & \beta & \beta \\
\beta & 0 & \beta_{X Y} & \beta \\
\beta & \beta_{X Y} & 0 & \beta \\
\beta_{B} & \beta & \beta & 0
\end{array}\right) .
\end{aligned}
$$

$\beta, \beta_{B}$ and $\beta_{X Y}$ are the dephasing rates. The decay of the exciton and biexciton is modeled by a single decay rate $\gamma$, which leads to the following equations of motion for the diagonal elements of the density matrix:

$$
\begin{aligned}
\varrho_{B B}(t) & =\varrho_{B B}(0) e^{-2 \gamma t} \\
\varrho_{X X}(t) & =\left[\varrho_{X X}(0)+\varrho_{B B}(0)\left(1-e^{-\gamma t}\right)\right] e^{-\gamma t} \\
\varrho_{Y Y}(t) & =\left[\varrho_{Y Y}(0)+\varrho_{B B}(0)\left(1-e^{-\gamma t}\right)\right] e^{-\gamma t} \\
\varrho_{G G}(t) & =1-\left[\varrho_{X X}(0)+\varrho_{Y Y}(0)+\varrho_{B B}(0)\left(2-e^{-\gamma t}\right)\right] e^{-\gamma t}
\end{aligned}
$$

The time $t=0$ corresponds to the time directly after each pulse.

From this, we can calculate the dynamics of all elements of the density matrix, in other words, of all populations and coherences. The FWM signal is theoretically extracted by analyzing the phase dependence of the polarization. In general, all polarizations have parts depending on different orders and combinations of the phases $\varphi_{i}$ of the pulses. The two-pulse FWM for coherence dynamics is given by the phase combination $\left(2 \varphi_{2}-\varphi_{1}\right)$, while the three-pulse FWM for the population dynamics is characterized by the phase combination $\left(\varphi_{3}+\varphi_{2}-\varphi_{1}\right)$ which model the heterodyning at $\left(2 \Omega_{2}-\Omega_{1}\right)$ and $\left(\Omega_{3}+\Omega_{2}-\Omega_{1}\right)$. This identifies the polarization of the FWM signal indicated by $p^{\text {FWM }}$. For the sake of simplicity, in the case of population dynamics we use $\tau_{12}=0$ ps to mimic the short time delay between the first two pulses. From the polarization the FWM signal $\mathcal{S}_{v v^{\prime}}$ is obtained by a Fourier transform at the selected frequency

$$
\mathcal{S}_{v v^{\prime}}=\left|\int_{0}^{\infty} p^{\mathrm{FWM}} e^{i \omega t} \mathrm{~d} t\right|_{\omega=\omega_{v}-\omega_{v^{\prime}}} .
$$

If the polarization $\alpha$ is not along one axis of the $\mathrm{QD}$, the signals are added according to the angle of the heterodyning (reference) beam $\alpha_{r}$ with $\mathcal{S}_{G X Y}=\cos ^{2}\left(\alpha_{r}\right) \mathcal{S}_{G X}+\sin ^{2}\left(\alpha_{r}\right) \mathcal{S}_{G Y}$.

In the FWM signal, charge fluctuations can play an important role leading to an inhomogeneous broadening via spectral wandering of individual transitions. This phenomenon induces a photon echo in FWM transients of single QDs, when probing the coherence [9-11]. The residual inhomogeneous broadening (i.e. up to several homogeneous linewidths) can be included in the calculations by multiplying the FWM-polarization with a Gaussian function [10] as follows:

$$
p^{\mathrm{FWM}} \rightarrow p^{\mathrm{FWM}} e^{-\frac{\left(t-\tau_{12}\right)^{2}}{2 \sigma^{2}}}
$$

For most cases the inhomogeneous broadening can be neglected. We only included it to model the data in Fig. 4 with $\sigma=67$ ps, which corresponds to an energetic broadening of $\hbar \sigma \approx 10 \mu \mathrm{eV}$.

\section{REFERENCES}

1. Y.-M. He, Y. He, Y.-J. Wei, D. Wu, M. Atatüre, C. Schneider, S. Höfling, M. Kamp, C.-Y. Lu, and J.-W. Pan, “On-demand semiconductor single-photon source with near-unity indistinguishability," Nature Nanotech. 8, 213 (2013).
2. Y. He, Y.-M. He, Y.-J. Wei, X. Jiang, M.-C. Chen, F.-L. Xiong, Y. Zhao, C. Schneider, M. Kamp, S. Höfling, C.-Y. Lu, and J.W. Pan, "Indistinguishable tunable single photons emitted by spin-flip Raman transitions in InGaAs quantum dots," Phys. Rev. Lett. 111, 237403 (2013).

3. S. Maier, P. Gold, A. Forchel, N. Gregersen, J. Mørk, S. Höfling, C. Schneider, and M. Kamp, "Bright single photon source based on self-aligned quantum dot-cavity systems," Optics Express 22, 8136 (2014).

4. F. Fras, Q. Mermillod, G. Nogues, C. Hoarau, C. Schneider, M. Kamp, S. Höfling, W. Langbein, and J. Kasprzak, "Multi-Wave Coherent Control of a Solid State Single Emitter," Nature Photon. 10, 155 (2016).

5. J. Kasprzak and W. Langbein, "Four-wave mixing from individual excitons: Intensity dependence and imaging," Phys. Status Solidi B 246, 820 (2009).

6. J. Kasprzak, B. Patton, V. Savona, and W. Langbein, “Coherent coupling between distant excitons revealed by twodimensional nonlinear hyperspectral imaging," Nature Photon. 5, 57 (2011).

7. M. Gschrey, A. Thoma, P. Schnauber, M. Seifried, R. Schmidt, B. Wohlfeil, L. Krüger, J.-H. Schulze, T. Heindel, S. Burger, A. Strittmatter, S. Rodt, and S. Reitzenstein, "Highly indistinguishable photons from deterministic quantum-dot microlenses utilizing three-dimensional in situ electron-beam lithography," Nature Comm. 6 (2015).

8. A. Vagov, V. M. Axt, and T. Kuhn, "Electron-phonon dynamics in optically excited quantum dots: Exact solution for multiple ultrashort laser pulses," Phys. Rev. B 66, 165312 (2002).

9. B. Patton, W. Langbein, U. Woggon, L. Maingault, and H. Mariette, "Time-and spectrally-resolved four-wave mixing in single CdTe/ZnTe quantum dots," Phys. Rev. B 73, 235354 (2006).

10. J. Kasprzak, S. Portolan, A. Rastelli, L. Wang, J. D. Plumhof, O. G. Schmidt, and W. Langbein, "Vectorial nonlinear coherent response of a strongly confined exciton-biexciton system," New J. Phys. 15, 055006 (2013).

11. Q. Mermillod, T. Jakubczyk, V. Delmonte, A. Delga, E. Peinke, J.-M. Gérard, J. Claudon, and J. Kasprzak, "Harvesting, coupling and control of single exciton coherences enabled by photonic trumpets," submitted (2016). 